\title{
ASSIMILATING MODELS AND DATA TO ENHANCE PREDICTIONS OF SHORELINE EVOLUTION
}

\author{
Joseph W. Long ${ }^{1}$ and Nathaniel G. Plant ${ }^{1}$
}

A modeling system that considers both long- and short-term process-driven shoreline change is presented. The modeling system is integrated into a data assimilation framework that uses sparse observations of shoreline change to correct a model forecast and to determine unobserved model variables and free parameters. Application of the assimilation algorithm also provides quantitative statistical estimates of uncertainty that can be applied to coastal hazard and vulnerability assessments. Significant attention is given to the estimation of four non-observable quantities using the data assimilation framework that utilizes only one observable process (i.e. ,shoreline change). The general framework discussed here can be applied to many other geophysical processes by simply changing the model component to one applicable to the processes of interest.

Keywords: data assimilation; shoreline change; sediment transport; morphology

\section{INTRODUCTION}

Shorelines represent the dynamic boundary separating beaches from the continual impact of waves, winds, surge, and tides. This boundary evolves over timescales of hours (e.g., changing tides or wave conditions) to decades (land subsidence, rising sea level, etc). The full spectrum of processes responsible for forcing evolution of the shoreline is not well understood, hindering the development of skillful predictive models. The need for such models, however, is becoming increasingly clear as coastal managers require information about shoreline evolution to plan construction setbacks, beachnourishment projects, and to assess coastal vulnerability to a variety of natural events.

Most existing studies of shoreline evolution consider long-term or short-term processes in isolation. When considering the former, the typical approach consists of determining the shoreline migration rate using a linear regression of historical shoreline position. Observations scattered about the best-fit regression line are considered noise. This type of analysis is intended to characterize historical response and is, essentially, a hindcast model of shoreline change. If predictions of future positions are required from this approach, they are extrapolated using the best-fit slope. There are a number of inherent problems in forecasting shoreline position with this method. This approach neglects acceleration of the shoreline-change rate and eliminates all short-term process-driven shoreline change. Moreover, because shoreline positions are often measured immediately after large storm events, the data may provide change rates that are biased high by neglecting natural beach recovery (e.g., Frazier et al. 2009).

Studies of short-term process-driven shoreline change attempt to focus more on annual and stormdriven change by assuming that the primary driver is the incident wave height (e.g., Miller and Dean 2004, Yates et al. 2009). Unfortunately, the exact relation between wave height and shoreline position is unclear. This leads to highly parameterized and calibrated models that are site-specific and do not account for temporal variations in the model free parameters. Moreover, the time horizon of coastal managers is dependent on the application at hand. Therefore, a model that considers both long- and short-term processes will provide greater flexibility in determining vulnerability assessments and project planning. Finally, assessment of coastal vulnerability and risk must always take into account some level of uncertainty. Unfortunately, the current numerical models can only provide information about hindcast model performance (i.e., spread/variability in best-fit parameters, etc.) and do not provide adequate information about forecast uncertainty.

Given the unknowns associated with predicting shoreline evolution, it is inevitable that as the prediction horizon increases, the model accuracy will degrade. Eventually, the uncertainty in the forecast will reach a point that renders it useless. One method to help 'guide' numerical models used for long-term predictions is to periodically update the forecast with available observations. This correction serves to re-initialize the modeled position. However, neither the model nor the data should be considered as exact. They are both methods that help estimate a particular geophysical state, but they both have limitations. Measurements suffer from instrument noise and offsets, and are spatially and/or temporally sparse. Models, on the other hand, must employ parameterizations of sub-grid-scale processes and often suffer from poor initial or boundary conditions. Therefore, the ideal approach to coastal-change forecasting is one that combines the inherent advantages of models and data to obtain accurate forecasts with the additional benefit of being able to quantify the uncertainty in the combined forecast.

${ }^{1}$ U.S. Geological Survey, St. Petersburg Coastal and Marine Science Center, 600 4th St. S., St. Petersburg, FL, 33701, USA 
This work represents an effort to devise a numerical model capable of forecasting combined longand short-term shoreline evolution and implementing it as part of a data assimilation framework for coastal change. The data assimilation scheme accomplishes three goals: 1) periodically correcting the modeled shoreline position with available observations to prevent forecasts from diverging from the true state; 2) estimating shoreline positions, rates, and model free parameters; and 3) providing a quantitative measure of the forecast uncertainty based on knowledge of the accuracy of both the model and the data. The numerical model and data assimilation strategy are documented in the second and third sections, respectively. We then present a demonstration of this method using an idealized model scenario followed by brief concluding remarks.

\section{SHORELINE-EVOLUTION MODEL}

Shoreline position, $X(t)$, is treated here as the summation of a position driven by long-term processes, $X_{l t}(t)$, and a short-term position, $X_{s t}(t)$, such that

$$
X(t)=X_{l t}(t)+X_{s t}(t) .
$$

The position of the shoreline, considering only long-term processes (such as sea-level rise), can be expressed with a typical linear model using a nearly constant change rate $\left(v_{l t}\right)$. With this, changes in the shoreline position are represented as

$$
X_{l t}(t+\Delta t)=X_{l t}(t)+v_{l t} \Delta t
$$

At this stage, we do not actually attribute the long-term shoreline movement to a particular process, nor do we perform a linear regression to determine the applicable change rate. Instead, we will use the combined model and available data to simultaneously determine the long- and short-term model states and parameters.

We assume that shorter-term variations in the shoreline position are driven by the incident wave height and use the equilibrium model approach of Plant et al. (1999). Equilibrium theory assumes that for a given wave height, there exists a shoreline position such that the beach would be in equilibrium (i.e., remain stationary with stationary wave forcing). Variations of this method have been applied by other studies of shoreline evolution (e.g., Miller and Dean 2004, Yates et al. 2009) when considering shoreline time series on the order of years. Of course, because the wave height varies continuously, the beach is constantly trying to move from one equilibrium state to another and may never reach equilibrium, depending on the rate at which the wave height changes. This method provides a description of the rate of shoreline movement, based on the present position of the shoreline and the natural equilibrium condition it is trying to achieve.

$$
\frac{d X_{S t}}{d t}=-a_{1} H(t)^{p}\left[X_{s t}(t)-\beta H(t)\right] \Delta t
$$

In Equation 3, $H(t)$ represents the time varying wave height and $\beta$ is a free parameter that linearly relates the wave height to the associated equilibrium shoreline position. The term in front of the brackets, $-a_{1} H(t)^{p}$, describes how fast the shoreline is able to move from the present position $\left(X_{s t}(t)\right)$ to the equilibrium position and is assumed to be related to wave height so that large waves move the shoreline position faster than small waves.

The combined model for shoreline position is then:

$$
X(t)=X_{l t}(t)+v_{l t} \Delta t+X_{s t}(t)-a_{1} H(t)^{p}\left[X_{s t}(t)-\beta H(t)\right] \Delta t
$$

The model is solved using a forward Euler finite-difference scheme with either a measured wave-height time series (hindcast) or a schematized/climatological wave-height time series (forecast). We use a time step of 0.1 months, and the model prediction is updated with the data assimilation filter described below at time steps when observations of shoreline position are available.

\section{DATA ASSIMILATION ALGORITHM}

The combination of modeled and observed shoreline position is achieved using the Kalman filter (Kalman 1960, Maybeck 1979). Here, the Kalman filter serves as an optimal data-processing algorithm that allows for a way to combine observed and modeled information about a physical state (i.e., shoreline position). The filter minimizes the variance of the error in the analyzed state (includes 
model forecast and observed state) and because it is a recursive filter, it is necessary to store prior information from only the most recent time step. Hence, it is a computationally efficient algorithm, which is essential for long-term forecasting. Observations of the state can be non-uniform in space and time and inferred from different types of instruments with different noise thresholds.

The general form of the Kalman filter is given by:

$$
\psi^{a}=\psi^{f}+K\left(d-M \psi^{f}\right)
$$

where $\psi$ is the physical state of interest and $\psi^{f}$ and $\psi^{a}$ represent the forecasted and analyzed state, respectively. The forecasted state corresponds to the value predicted by the model at that time step, while the analyzed state represents a state "corrected" by the data available at that time step. The quantity in parentheses is the innovation, or the difference between the observation, $d$, and the corresponding modeled state, $M \psi^{f}$. Note that the filter does not require that the observed state and the forecasted state be the same, only that they are linearly related by $M$. The innovation is weighted by the Kalman gain, which is computed using the following equation:

$$
K=C^{f} M^{T}\left(M C^{f} M^{T}+C_{d}\right)^{-1}
$$

In the above, the superscript $T$ represents the transpose of the matrix. Therefore, the innovation is weighted according to the error covariance of the predicted state, $C^{f}$, and the observed state, $C_{d}$. For small values of $C_{d}$ (very accurate measurements), the value of $K$ tends toward unity, and the analyzed state becomes equal to the observation. Alternately, when the observations are noisy or inaccurate and $C_{d}$ is large, the forecast will not be influenced by the data and will remain equal to the value forecasted by the model. After the forecast has been updated with available data, the error covariance of the analyzed state (the state that includes information from both the model and the data) is updated by

$$
C^{a}=(I-K M) C^{f}
$$

where $I$ is the identity matrix. This provides the quantitative value of the uncertainty that remains in the analyzed state estimate.

The Kalman filter requires that the physical state be described by a linear model. Therefore, we will assume that the values of $a_{1}$ and the exponent $p$ in Equations 3 and 4 will be constant with values inferred from previous studies (e.g., Plant et al. 1999, $p=3 ; a_{1}=1$ ). We make use of the fact that the observable does not have to be the exact state of interest to expand the analysis in vector form so that

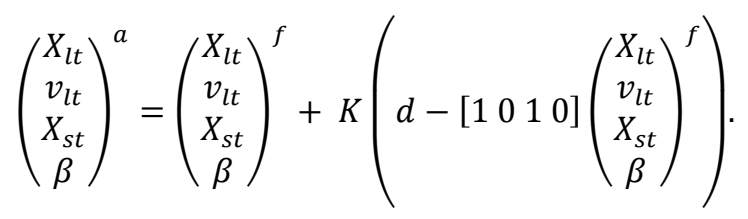

Here, our observable is the combined shoreline position that would be measured with a discrete morphological survey $(X(t))$. This observation is related to our four states of interest, which include the separated short-term and long-term shoreline positions, long-term migration rate, and the model parameter, $\beta$. At each time step when data are available, these quantities will be updated and then used to forecast to the next time step. We also track the error covariance of each of the corrected variables, which provides a measure of uncertainty in the analysis.

\section{IDEALIZED CASE STUDY}

As an initial test, we implement the model-data assimilation algorithm using an idealized case study. This is designed to demonstrate an application of this approach and illustrate the ability of this method to estimate multiple variables/parameters from one related but separate observable. A 13-year wave-height time series is constructed that contains seasonal variations in wave energy along with some characteristic noise (Figure 1). Given this time series, the "true" shoreline position is determined using Equation 4 with $v_{l t}=0.6, p=3, a_{1}=1$, and $\beta=1$. The 'data' to be used in the assimilation process are then sub-sampled, annually, from this time series of shoreline position, and normally distributed noise is added to each yearly observation. Note that this sub-sampling interval does not resolve the seasonal, short-term shoreline modulation. 


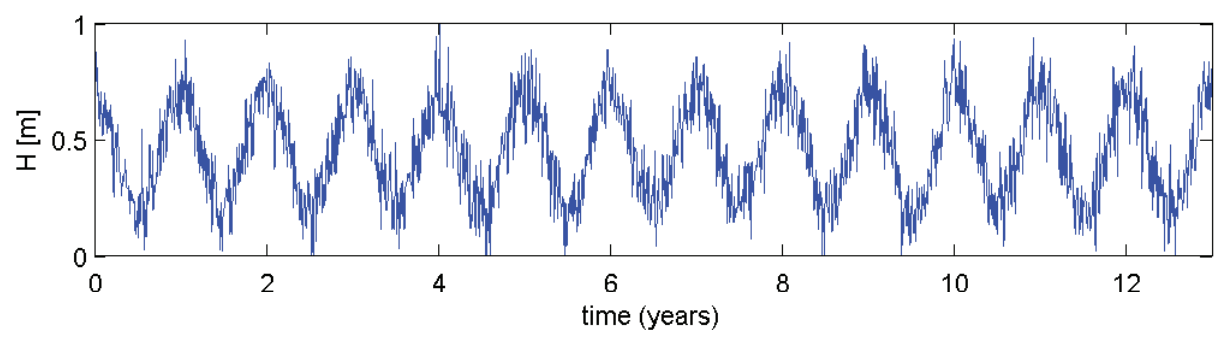

Figure 1. Synthetic wave-height time series used in shoreline-evolution model.

We then model the shoreline position by choosing incorrect values for the model parameters and later allow the values to be updated and estimated by the assimilation process. Here, we initialize the model with the following: $v_{l t}=0$ and $\beta=0$. Hence, we assume there is no long-term component of shoreline evolution and that the short-term movement is unrelated to the equilibrium position. Then, at each time step in the model when data are available, we compute the Kalman gain and allow the filter to update the modeled shoreline position and, in particular, the four specific quantities of interest $\left(X_{l t}, v_{l t} X_{s t}, \beta\right)$.

The time history of the shoreline position and individual quantities are given in Figure 2. Clearly, the model, initialized with incorrect physical conditions, would have given an erroneous forecast of the shoreline position. The Kalman filtering approach, however, was able to converge on the correct shoreline position within a two-year period. The filtering routine was also able to detect the long-term shoreline movement from the data and to determine the actual long-term migration rate, despite the fact that it had been assumed to be zero at the beginning of the simulation period. Likewise, both the shortterm shoreline position and the relation between the wave height and equilibrium shoreline position were identified. The results indicate a decreasing uncertainty with time when data are available to keep the model on track. Once values converged to the true values, the levels of uncertainty also converged to the minimum levels of uncertainty which correspond to the error estimates provided to the Kalman filter for the model and data.

\section{SUMMARY}

This paper represents the first steps toward developing a forecasting system consisting of a shoreline-evolution model that incorporates a range of long- and short-term processes and a Kalman filter data assimilation scheme. Assuming that the dominant physics are represented in the model formulation, the predictor-corrector cycle of the Kalman filter will update modeled quantities, including free parameters. It is expected that if these quantities change with time (i.e., change of longterm migration rate), the filter will identify this change, unlike typical linear-regression analysis of historical data. The case study included here showed the ability to estimate four model quantities with the assimilation of only one variable, which itself was not one of the quantities being estimated. The algorithm was able to separate short- and long-term processes despite our having initialized the prediction with inaccurate model parameters. We suggest that data assimilation is an under-utilized tool in coastal/nearshore science and that it is essential when forecasting morphological conditions, especially over long timescales. 

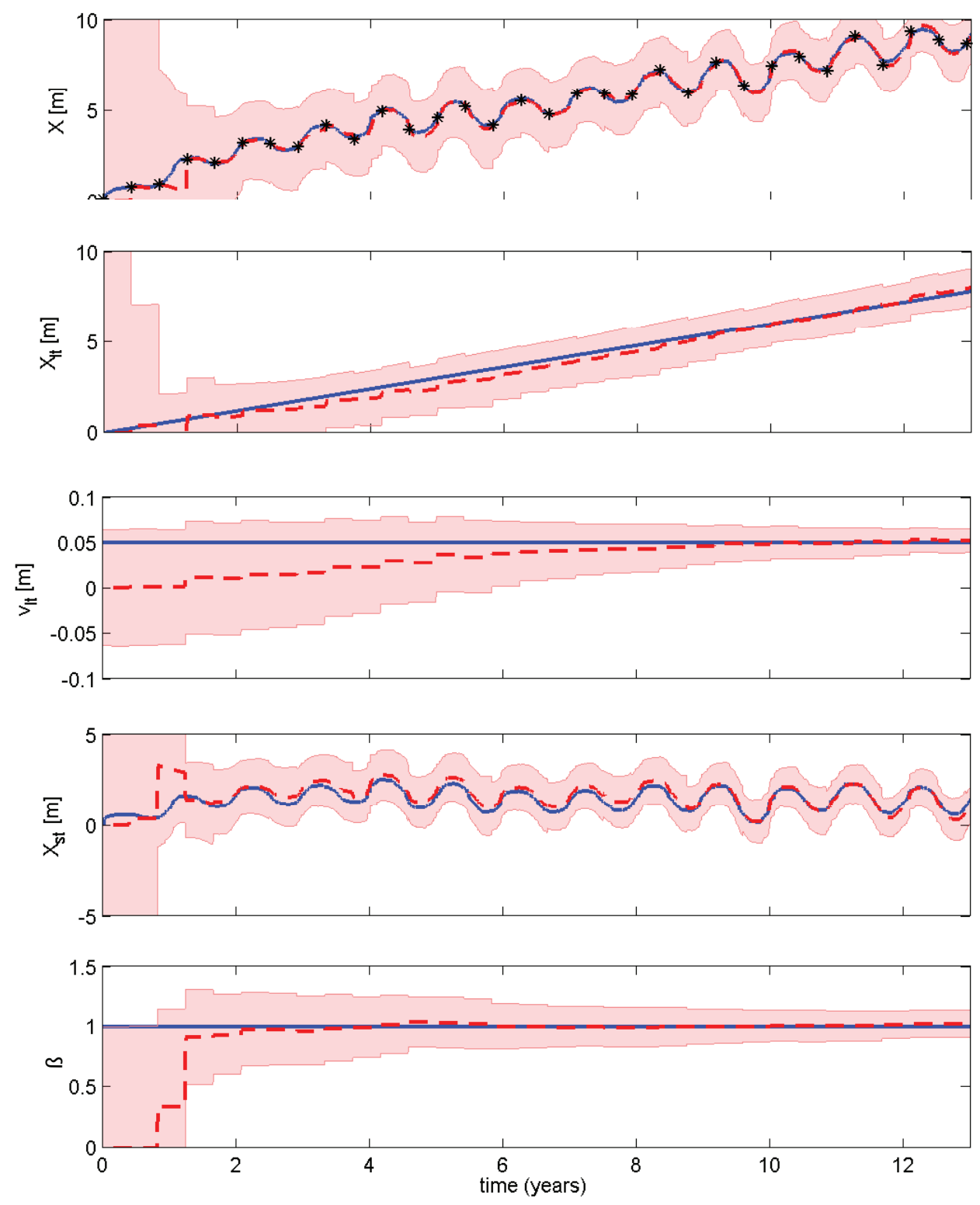

Figure 2. Results from the model-data assimilation algorithm. (top) Total shoreline position comparing "true" (blue) and modeled (red) results and data (asterisks) used in the assimilation process. (middle-top) Long-term shoreline change rate. (middle-bottom) Short-term shoreline position. (bottom) Free model parameter $(\beta)$ relating instantaneous wave height to an equilibrium shoreline position.

\section{ACKNOWLEDGMENTS}

This work was funded by the Mendenhall Postdoctoral Program at the U. S. Geological Survey.

\section{REFERENCES}

Frazer, L., T. Anderson, and C. Fletcher. 2009. Modeling storms improves estimates of long-term shoreline change, Geophysical Research Letters, 36(20), L20404.

Kalman, R. 1960. A new approach to linear filtering and prediction problems, Journal of basic Engineering, 82(1), 35-45.

Maybeck, P. 1979. Stochastic models, estimation, and control, volume 141 of Mathematics in Science and Engineering, pp. 1-16. 
Miller, J. and R. Dean. 2004. A simple new shoreline change model, Coastal Engineering, 51(7), 531556.

Plant, N. G., R. A. Holman, M. H. Freilich, and W. A. Birkemeier. 1999. A simple model for interannual sandbar behavior, Journal of Geophysical Research, 104(C7), 15,755-15,776.

Yates, M., R. Guza, and W. O'Reilly. 2009. Equilibrium shoreline response: Observations and modeling, Journal of Geophysical Research, 114. 